\section{Список литературы:}

1. Репин Д.В., Репина Н.В. Анализ эффективности использования научно-исследовательской деятельности для формирования экологических знаний студентов // Биоэкологическое краеведение: мировые, российские и региональные проблемы / отв. ред. С.И. Павлов. Самара: СГСПУ, 2018. С. 313-315.

2. Репин Д.В., Семенов Л.В. Повышение уровня географических знаний обучающихся с помощью логических опорных схем // Природные и социальные экосистемы. Чебоксары: Чуваш. гос. пед. ун-т, 2019. C. $126-129$.

3. Репин Д.В., Якунина А.А. Формирование экологической культуры обучающихся во внеурочной деятельности // Природные и социальные экосистемы. Чебоксары: Чуваш. гос. пед. ун-т, 2019. С. 129-133.

4. Бирина О.В. Понятие успешности обучения в современных педагогических и психологических теориях // Фундаментальные исследования. 2014. № 8, ч. 2. C. $438-443$.

5. Вилюс В.К. Психологические механизмы мотивации человека. М.: Проспект, 2013. 170 с.

6. Курапова Т.Ю. Психологические факторы успешности обучения школьников // Ученые записки ЗабГГПУ. 2011. № 5 (40). С. 223-225.
7. Лоренц Д.В. Креативная педагогика на примере дисциплины: учеб.-метод. пособие. М.: Инфра-М, 2018. $252 \mathrm{c}$.

8. Шатунова Е.Ю. Создание ситуации успеха в учебно-воспитательном процессе // Концепт. 2014. T. 12. C. 206-210.

9. Щукина Г.И. Активация познавательной деятельности в учебном процессе. М.: Просвещение, 2015. $144 \mathrm{c}$.

10. Лапыгин Ю.Н. Методы активного обучения: учебник и практикум для вузов. М.: Юрайт, 2019. $248 \mathrm{c}$.

11. Шаталов В.Ф. Куда и как исчезли тройки: из опыта работы школ г. Донецка. М.: Педагогика, 1979. $134 \mathrm{c}$.

12. Шаталов В.Ф.Учить всех, учить каждого // Педагогический поиск. М.: Педагогика, 1989. 560 с.

13. Шаталов В.Ф. Эксперимент продолжается. Донецк: Сталкер, 1998. 396 с.

14. Калмыкова Н.В., Петряева С.Ф. Опорный конспект как один из способов представления учебной информации // Молодой ученый. 2015. № 11.1 (91.1). C. 53-58.

\title{
THE USE OF LOGICAL REFERENCE SCHEMES \\ AS A MEANS OF INCREASING THE LEVEL OF STUDENTS' ENVIRONMENTAL KNOWLEDGE
} (C) 2019

Repin Denis Vladimirovich, candidate of biological sciences, associate professor of Bioecology and Chemistry Department I. Yakovlev Chuvash State Pedagogical University (Cheboksary, Russian Federation)

Repina Nadezhda Vasilyevna, candidate of biological sciences, lecturer Cheboksary Cooperative Technical School of Chuvashpotrebsoyuz (Cheboksary, Russian Federation)

Abstract. This paper describes the results of experimental work on the use of logical support circuits as a means of increasing the environmental knowledge of students of technological, economic and graphic arts departments of I. Yakovlev Chuvash State Pedagogical University. The research was carried out from February 2019 to May 2019. During the experiment, students of Technology and Economics Department were selected as a control group, and students of Art and Graphics Department as an experimental group. In the control group, training was carried out without the use of logical support schemes. In the experimental group, the educational process used logical support schemes. The determination of the initial level of ecological knowledge of students of both groups revealed close values of this parameter. After the experiment on the logical reference circuits use in the educational process, a repeated testing was carried out. Its results in both groups were higher than at the initial stage of the experiment. At the same time, when comparing the data of the control and experimental groups, significant differences were revealed. The students who used logical support schemes had significantly higher test scores than the students of the control group.

Keywords: logical reference circuits; reference signals; V.F. Shatalov; ecology; environmental knowledge; student; experimental work; testing; visual aids; abstract thinking; note taking; theoretical material; studying process; I. Yakovlev Chuvash State Pedagogical University.

\section{ТЕХНОЛОГИЧЕСКИЙ ПРОЦЕСС ВНЕАУДИТОРНОЙ ИНДИВИДУАЛИЗАЦИИ ПОДГОТОВКИ ЗАРУБЕЖНЫХ МАГИСТРОВ УПРАВЛЕНИЯ ОБРАЗОВАНИЕМ}

Рыблова Алла Николаевна, доктор педагогических наук, профессор кафедры лингводидактики Московский государственный лингвистический университет (2. Москва, Российская Федерация)

Кан Цюнцюн, аспирант факультета педагогического образования

Московский государственный университет им. М.В. Ломоносова (2. Москва, Российская Федераиия)

Аннотащия. В статье ставится и решается проблема технологичной организации внеаудиторной индивидуальной деятельности зарубежных магистрантов. Технологичность индивидуализированного образователь- 
ного процесса в магистратуре, развивающаяся на основе реализации возможностей информационнотелекоммуникационных технологий, обеспечивает более быстрое и качественное овладение зарубежными магистрантами профессиональными знаниями, умениями и навыками. Информационные библиотечные ресурсы играют значительную роль в их становлении, предоставляют большие дидактические возможности для индивидуализированной подготовки зарубежных магистров управления образованием, недостаточно хорошо владеющих русским языком и, как результат, нуждающихся в тьюторской поддержке во внеаудиторное время для переработки профессионально значимой научной информации. Авторы представляют теоретикометодологическое обоснование и результаты моделирования и апробации технологии внеаудиторной индивидуализации подготовки зарубежных магистров управления образованием под руководством сотрудника библиотеки с помощью комплекса информационных библиотечных ресурсов. Эта технология предусматривает взаимосвязанную образовательную и научно-исследовательскую деятельность зарубежного магистранта и консультативную деятельность сотрудника библиотеки, реализуемую во внеаудиторное время и направленную на индивидуальную переработку профессионально значимой научной информации в профессионально заданных ситуациях с помощью комплекса информационных библиотечных ресурсов, используемых в качестве средств педагогической коммуникации для достижения профессиональных целей. Комплекс информационных библиотечных ресурсов включает: печатные фонды, электронные каталоги, поисковые платформы и электронные базы данных и учитывает направление подготовки зарубежных магистрантов. Технологический процесс внеаудиторной индивидуализации предусматривает алгоритм взаимосвязанных действий сотрудника библиотеки и зарубежного магистранта по проектированию, организации, реализации, контролю, оцениванию и диагностике образовательных результатов и затруднений внеаудиторной индивидуальной научной деятельности. Определены организационно-педагогические условия для эффективной реализации авторской технологии и разработан диагностический инструментарий, включающий тесты и критерии диагностики уровня овладения комплексом информационно-библиотечных ресурсов. Смоделированная технология обеспечивает индивидуальную информационную, методическую поддержку каждому зарубежному магистранту и улучшает качество их научно-исследовательской деятельности, реализуемой в виртуальном образовательном пространстве библиотек.

Ключевые слова: технологический процесс; подготовка зарубежных магистров управления образованием; технология внеаудиторной индивидуализации; комплекс информационных библиотечных ресурсов; библиотекарь; индивидуальный контроль.

\section{Постановка проблемы}

За последние два десятилетия появилось много исследований, посвященных технологичной организации индивидуального обучения вследствие появления реальной возможности широкого использования информационных технологий в образовательном процессе системы уровневого высшего образования. В процессе теоретико-методологического анализа научной литературы по проблемам индивидуализации обучения мы пришли к пониманию этого непростого в реализации вида обучения, который предусматривает учет индивидуальных потребностей и индивидуально-психологических особенностей обучающихся при постановке целей, выборе содержания и времени на его изучение, отборе методов и средств обучения, способствующих раскрытию индивидуальности каждого обучающегося.

По мнению ряда исследователей (И.М. Осмоловская [1], А.Н. Рыблова [2], Г.К. Селевко [3], И.Э. Унт [4] и др.), технология индивидуализации обучения это спроектированный, организуемый, реализуемый и диагностируемый в аудиторное и внеаудиторное время образовательный процесс, при котором выбор целей, отбор содержания, методов, средств и сроков обучения обусловливаются индивидуальными потребностями и особенностями обучающихся. Проблемы, которые возникают в процессе обучения в магистратуре, заключаются в большей мере в том, каким образом более быстро и качественно организовать магистрантам самостоятельную научно-исследовательскую деятельность во внеаудиторное время.

Российские и китайские ученые предлагают разные варианты решения этой проблемы. Т.М. Ковалёва предложила модель тьюторского сопровождения, в которой роль тьютора простирается от лекций в классе до сопутствующего консультирования вне класса [5]. Е.И. Зарипова считает, что индивидуальная образовательная программа (ИОП) студента является средством индивидуализации магистерской подготовки. Она обобщила методы создания ИОП с помощью тьюторской практики в российских вузах [6]. Китайский учёный Лю Сяньцзюнь выдвинул идею создания позиции «потенциального тьютора»в университете [7]. Задача потенциального тьютора состоит в том, чтобы помочь студентам раскрыть их потенциал, спланировать обучение в университете в соответствии с их интересами и личностными характеристиками посредством систематического руководства и индивидуализированного обучения во внеаудиторное время.

Реализуя в нашем теоретико-экспериментальном исследовании идею индивидуального тьюторства в образовательном процессе, мы предлагаем инновационный подход, предусматривающий руководство библиотекарем индивидуализированной образовательной и научно-исследовательской деятельностью зарубежных магистрантов во внеаудиторное время с помощью комплекса информационных библиотечных ресурсов. Технологичность индивидуализированного образовательного процесса в магистратуре, развивающаяся на основе реализации возможностей информационно-телекоммуникационных технологий, обеспечивает более быстрое и качественное овладение профессиональными знаниями, умениями и навыками. Информационные библиотечные ресурсы играют значительную роль в их становлении.

\section{Методология теоретико- \\ экспериментального исследования}

Смоделированная нами на основе технологического подхода технология опирается на научные исследования отечественных и зарубежных ученых (Б. Блум [8], Н. Гронлунд [9], А.Н. Рыблова [10], 
Рыблова А.Н., Кан Ц.

Пи Ляньшен [11; 12], Ву Фати [13] и др.) открывает большие возможности для индивидуализированной подготовки зарубежных магистров управления образованием, недостаточно хорошо владеющих русским языком и, как результат, нуждающихся в тьюторской поддержке во внеаудиторное время для переработки профессионально значимой научной информации $[14 ; 15]$.

Технология внеаудиторной индивидуализации (далее - ТВнИнд) подготовки магистров управления образованием предусматривает алгоритм взаимосвязанной образовательной и научно-исследовательской деятельности зарубежного магистранта и консультативной деятельности сотрудника библиотеки, реализуемой во внеаудиторное время и направленной на индивидуальную переработку профессионально значимой научной информации в профессионально заданных ситуациях с помощью комплекса информационных библиотечных ресурсов (далее - КИБР), используемых в качестве средств педагогической коммуникации для достижения профессиональных целей [16]. Выбранный нами комплекс информационных библиотечных ресурсов включает: печатные фонды, электронные каталоги, поисковые платформы и электронные базы данных и учитывает направление подготовки зарубежных магистрантов.

В процессе внеаудиторного обучения библиотекарь использует современные информационные и коммуникационные технологии для создания платформы дистанционного обучения и применяет модель «контроль - самоорганизация» для проведения индивидуализированного обучения на платформе. Эта модель предусматривает, что в условиях хорошо продуманного управления процессом учения, зарубежные магистранты достигают профессионально значимых целей посредством самоорганизации образовательного процесса. В этом процессе сотрудник библиотеки не должен играть роль просто источника профессионально значимой информации, а может быть помощником и консультантом.

Технологический процесс предусматривает алгоритм взаимосвязанных действий сотрудника библиотеки и каждого магистранта по проектированию, организации, реализации, оценивании и диагностике достижений и затруднений внеаудиторной индивидуальной образовательной и научно-исследовательской деятельности. Для его реализации широко применяются интерактивные и проектные методы обучения.

\section{1. Проектирование внеаудиторной \\ индивидуальной деятельности \\ зарубежных магистрантов}

При проектировании внеаудиторной индивидуальной деятельности зарубежных магистров управления образованием необходим анализ индивидуальных профессиональных потребностей каждого обучающегося и их индивидуально-психологических особенностей. Кроме того, следует определить индивидуальные профессионально значимые цели. Затем нужно осуществить индивидуальный отбор КИБР для поиска профессионально значимой научной информации.

Анализ индивидуальных профессиональных потребностей. Сотрудник библиотеки должен проанализировать профессиональные потребности каждого зарубежного магистранта, так как анализ потребностей является основным компонентом индивидуальной образовательной деятельности обучающегося и его результаты значительно влияют на качество его самостоятельной научно-исследовательской деятельности. Для этого сотрудник библиотеки сначала должен провести опрос обучающихся с помощью интервью или разного вида опросников, анкет и др. Затем проанализировать полученные данные и на их основе принять решение: уменьшить или увеличить объем психолого-педагогического воздействия. Всё это поможет убедиться в том, что проектируемая образовательная и научно-исследовательская деятельность является теоретически обоснованной и методически применимой в рамках реализуемой рабочей программы. Затем проходит оценка возможности осуществления этих потребностей в имеющихся образовательных условиях во внеаудиторное время.

Анализ индивидуально-психологических особенностей зарубежных магистрантов предусматривает учет особенностей темперамента, воли, характера и др. обучающихся, т.е. включает изучение психологических и физиологических факторов, влияющих на внеаудиторный образовательный процесс. Как известно, образовательная деятельность основывается на уровне подготовки студентов, поэтому при проектировании необходимо понимать, какие есть у обучающихся знания и умения по той или иной дисциплине, а также их отношение к ней. Большую роль при этом играет стиль обучения. Стиль обучения это определённый способ обучения и тенденция в обучении, которые обучающиеся проявляют в долгосрочной учебной деятельности [17]; это описание поведения и отношений, которые могут определять предпочтительные методы обучения студента [18]. Обучающиеся с различными стилями обучения будут различаться в учебном поведении и стратегиях образовательного процесса. Только на основе полного понимания исходного уровня знаний и стиля обучения могут быть определены цели обучения, разработаны этапы и формы внеаудиторной научно-исследовательской деятельности магистрантов, поставлены задачи, отвечающие их профессиональным потребностям и индивидуально-психологическим особенностям.

Определение профессионально значимых иелей обучения предусматривает описание конкретных результатов образовательной и научно-исследовательской деятельности, которых каждый студент должен достичь после её завершения. Поскольку зарубежные магистранты различаются по личностным качествам, потребностям в обучении, мотивации к обучению и исходным уровнем подготовки, мы считаем, что должны быть разработаны дифференцированные цели обучения, т.е. есть для обучающихся с различными характеристиками устанавливаются разные ожидаемые результаты. Библиотекарь сначала должен создать детальную структуру целей обучения, а затем в процессе внеаудиторного обучения генерировать дифференцированные цели обучения посредством установления минимальных целей и дифференцирования задач в соответствии с индивидуальными характеристиками обучающихся. Минимальная цель - это цель, которую должны достичь все зарубежные магистранты. Она устанавливается библиотекарем на основании соответствующих документов и среднего уровня подготовки студентов. Когда магистрант с более высоким уровнем подготовки достигает минимальной цели, библиотекарь может увеличить объем и сложность учебного задания в 
рамках учебных задач, установленных рабочей программой.

Индивидуальный отбор КИБР для поиска профессионально значимой научной информации. Выбор источника профессионально значимой информаци нередко вызывает значительные затруднения у зарубежных магистрантов первого года обучения. Поэтому выбор КИБР можно предоставить каждому студенту исходя из его индивидуальных предпочтений и существующих технических возможностей. Задача сотрудника библиотеки при этом заключается в консультативной поддержке и разъяснении возникающих затруднений в процессе поиска и переработки профессионально значимой информации.

\section{2. Организаичи внеаудиторной индивидуальной деятельности зарубежных магистрантов}

Для организации внеаудиторной индивидуальной подготовки зарубежных магистров управления образованием необходимо создание сетевой платформы. Кроме того, следует осуществить выбор профессионально значимых ситуаций и определить количество и время проведения индивидуальных консультаций библиотекаря. Сотрудник библиотеки предъявляет образовательные ресурсы с помощью КИБР и объясняет специфику работы службы поддержки.

Организационный процесс внеаудиторной индивидуализации - это процесс построения сетевой образовательной среды. Сетевая образовательная среда является платформой поддержки обучения и сочетанием различных факторов, которые воздействуют на обучающихся и способствуют их обучению [13]. Мы считаем, что основными компонентами сетевой образовательной среды являются сетевые платформы обучения; сотрудники библиотеки и магистранты; технология их совместной внеаудиторной образовательной и научно-исследовательской деятельности; образовательные ресурсы и службы поддержки с помощью КИБР.

Сетевая платформа обучения - это основной компонент среды электронного обучения, который является не только каналом распространения образовательных ресурсов, но и средством коммуникации библиотекаря и магистранта. Поэтому библиотекарь должен сначала создать подходящую учебную платформу, обладающую базовыми функциями, такими как управление образовательными ресурсами, сетевое взаимодействие и поддержка инструментов обучения. С развитием технологий к настоящему времени появилось множество программ с открытым исходным кодом для онлайн-обучения и управления учебными программами, наиболее известными из которых являются Sakai, Moodle, Blackboard и т.д. Эти программы не только обеспечивают регулярные учебные модули, коммуникационные модули, но также обладают мощными функциями статистического анализа. Сотрудник библиотеки может использовать эти функции, чтобы диагностировать и сохранить личные данные об уровне знаний и умений магистрантов. Понимая особенности и технологии построения многих платформ, библиотекарь может создавать соответствующие платформы для обеспечения консультации в соответствии с индивидуальными потребностями зарубежных магистрантов управления образованием в профессионально заданных ситуациях.
Внеаудиторная образовательная и научно-исследовательская деятельность является совместной деятельностью сотрудника библиотеки и каждого магистранта для выполнения конкретных целей обучения. Здесь зарубежные магистранты могут самостоятельно планировать свое обучение в соответствии с образовательными ресурсами и учебными инструментами, предоставляемыми библиотекарями. В основе самостоятельной научно-исследовательской деятельности лежит вовлечение студентов в процесс обучения, что хорошо сказывается на развитии их способности к самостоятельному мышлению. Сотрудник библиотеки для организации индивидуальной поисково-исследовательской деятельности магистратов должен обратить внимание на следующие аспекты: прежде всего содержание курса должно стимулировать интерес к обучению; образовательные ресурсы должны быть структурированы для того, чтобы облегчить доступ студентов к ним; поддержка должна быть оказана каждому обучающемуся в любое время процесса самообучения.

Образовательные ресурсы являются теми информационными инструментами, которые зарубежные магистранты могут использовать для обучения, в том числе различные виды учебников, справочных материалов, упражнений и др. Разработка таких ресурсов осуществляется на основе индивидуальных профессиональных потребностей в образовательной и научно-исследовательской деятельности и должна рассматриваться с точки зрения видов и функций ресурсов. Виды ресурсов, которые часто используются в образовательном процессе, включают в себя текст, изображения, анимацию, аудио, видео и т.д. Функции ресурсов предусматривают действия по предъявлению учебных материалов, подробное объяснение, симулятор ситуаций, пробуждение воображения, практику и оценку. На практике различные виды ресурсов могут быть интегрированы для достижения наилучших индивидуальных образовательных результатов.

В настоящее время некоторые организации разработали систематические стандарты оценки разработки ресурсов. Например, Комитет по разработке современных ресурсов дистанционного образования Министерства образования Китая опубликовал документ «Технические спецификации для создания современных ресурсов дистанционного образования», в которых были четко указаны технические нормы текстов, изображений, аудио, видео, анимации и других материалов, используемых в дистанционном образовании. Согласно этому документу библиотекарь при организации внеаудиторной индивидуальной поисково-исследовательской деятельности магистрантов должен обращать внимание на такие технические показатели, как организация, передача и представление таких образовательных ресурсов.

Служба поддержки - это служба, предоставляемая обучающимся в среде онлайн-образования, включая службу индивидуальной поддержки библиотекарями и управленческой поддержки. Служба поддержки сотрудником библиотеки отражена в справочной службе, предоставляемой каждому магистранту для поиска и предъявления профессионально значимой научной информации, например, обсуждение и общение со студентами, ответы на вопросы, регулирование совместной образовательной и научно-исследовательской деятельности, предоставление 
Рыблова А.Н., Кан Ц.

студентам конкретных рекомендаций по самоорганизации внеаудиторного обучения. Служба управленческой поддержки отражена в справочной службе, предоставляемой библиотекарем студентам на учебной платформе, например, техническая помощь, консультации и т.д.

\section{3. Реализачия зарубежными магистрантами внеаудиторной индивидуальной деятельности \\ под руководством сотрудника библиотеки}

При реализации зарубежными магистрантами внеаудиторной индивидуальной поисково-исследовательской деятельности сотрудник библиотеки осуществляет отправку образовательных ресурсов КИБР на сетевую платформу. После этого обучающиеся индивидуально используют рекомендуемые ресурсы. При этом осуществляются индивидуальные коммуникации библиотекаря и каждого зарубежного магистранта в дистанционной форме. Сотрудник библиотеки регулирует индивидуальную поисковоисследовательскую деятельность каждого студента (не более трех магистрантов день). Для повышения эффективности внеаудиторного индивидуализированного обучения следует применять информационно-методический инструментарий, предусматривающий, прежде всего, разработку учебно-методического комплекса (УМК) по конкретной дисциплине. Такой комплекс был модернизирован нами по дисциплине «Информатизация образовательного процесса» для магистрантов 1 курса по направлению «Менеджмент», профилю «Управление образованием». В нем были предусмотрены функции сотрудника библиотеки для индивидуальной организации самостоятельной подготовки зарубежных магистрантов [19].

При возникновении каких-либо проблем во время внеаудиторной образовательной и научно-исследовательской деятельности, каждый студент может общаться с библиотекарем через сетевую платформу и получать необходимую информационную и методическую поддержку. Сотрудник библиотеки может получать информацию о темпе и качестве внеаудиторной научно-исследовательской деятельности каждого зарубежного магистранта по поиску и переработке профессионально значимой научной информации с помощью индивидуальных дистанционных коммуникаций, консультаций или др. Для студентов с более быстрым прогрессом в обучении библиотекарь может продолжать отправлять дополнительные более объемные и сложные образовательные ресурсы, чтобы направлять их к более глубокому освоению материала. Когда студент завершает выполнение задания, библиотекарь может организовать общение и совместную деятельность в виртуальном чате и обсудить междисциплинарные связи изучаемой дисциплины с профессиональными дисциплинами, изучаемыми зарубежными магистрантами с тем, чтобы показать им практическую значимость для будущей профессии.

В процессе общения с каждым обучающимся библиотекарь может дополнительно документировать индивидуальные характеристики и достижения каждого студента, анализировать их стиль обучения, разделить магистрантов с аналогичными характеристиками на группы и уровни и разрабатывать индивидуальные образовательные маршруты / траектории внеаудиторной научно-исследовательской деятельности. Это, несомненно, будет способствовать более быстрому и качественному становлению у зарубежных магистрантов умений и навыков научно-исследовательской деятельности.

\section{4. Индивидуальньй контроль, оценивание и диагностика достижений и затруднений во внеаудиторной индивидуальной} деятельности зарубежных магистрантов

Для проведения индивидуального контроля, оценивания и диагностики индивидуальных достижений и затруднений каждого зарубежного магистранта управления образованием предусмотрено диагностическое, формирующее и итоговое тестирование и анкетирование. Кроме того, осуществляется контроль и оценивание деятельности библиотекаря со стороны студентов и руководителей образовательной организации. Затем проводится индивидуальная коррекция образовательных результатов и диагностика проблем использования КИБР.

Основная цель контроля и оценивания заключается в предварительной, текущей и заключительной диагностике и коррекции образовательного процесca, проблем реализации учебной программы, а также в дальнейшем понимании индивидуальных профессиональных потребностей зарубежных магистрантов. Мы считаем, что контроль, оценивание и диагностика касается студентов и библиотекарей на протяжении всей учебной программы.

Для контроля, оценивания и диагностики достижений и затруднений студентов должен быть также разработан диагностический инструментарий, включающий тесты, анкеты, критерии, эталоны объема и правильного выполнения заданий и операций установленного объема за определенное время. Мы разработали следующие критерии трехуровневой диагностики уровня владения КИБР:

- способность определять характер и объём профессионально значимой научной информации исходя из профессионально значимых целей;

- способность эффективно получать доступ и перерабатывать профессионально значимую научную информацию;

- способность правильно оценивать профессионально значимую научную информацию и информационные источники, интегрировать их в собственную систему знаний и конструировать новую / уникальную информацию;

- способность эффективно управлять и обмениваться профессионально значимой научной информацией;

- способность самостоятельно использовать профессионально значимую научную информацию в профессионально заданных ситуациях [20].

Оценивание зарубежных магистрантов включает диагностическое, формирующее и итоговое оценивание. Диагностическое оценивание затрагивает анализ знаний, навыков и индивидуально-психологические особенности поведения обучающихся до начала образовательного процесса. Формирующее оценивание представляет собой оценивание, проводимое в ходе образовательного процесса для того, чтобы своевременно обнаружить индивидуальные проблемы переработки профессионально значимой научной информации в профессионально заданных ситуациях. Формирующее оценивание может быть реализо- 
вано посредством наблюдения за учебным поведением каждого зарубежного магистранта в процессе общения с библиотекарем, в рамках группового взаимодействия и во время индивидуального выполнения заданий, тестов и т.д. Итоговое оценивание проводится после завершения темы, раздела, курса и др., чтобы индивидуально диагностировать достижение образовательных результатов и затруднения каждого студента. Таким образом, в процессе оценивания зарубежных магистрантов, библиотекарь может обнаружить проблемы в индивидуальном образовательном процессе, своевременно исправить их, при необходимости скорректировать профессионально значимые цели, тем самым делая процесс обучения более технологичным.

Так как сотрудник библиотеки пока не закреплен официально в роли консультанта и тьютора внеаудиторного индивидуализированного образовательного процесса в магистратуре, контроль и оценивание такой деятельности библиотекаря необходимы для совершенствования его инновационной педагогической функции. Обучающимся предлагается индивидуально оценить своевременность и качество информационной и методической поддержки их поисковоисследовательской деятельности. Они могут оценивать своих библиотекарей с точки зрения объема и новизны предлагаемых образовательных ресурсов, разнообразия форм индивидуализированной образовательной и научно-исследовательской деятельности, службы поддержки и т.п. Оценивание результатов инновационной индивидуализированной консультативной и тьюторской деятельности сотрудников библиотеки со стороны руководителей образовательных организаций высшего образования может содействовать улучшению педагогических и управленческих навыков.

\section{Результаты исследования}

Экспериментальное исследование эффективности технологии внеаудиторной индивидуализации подготовки зарубежных магистров управления образованием было проведено на факультете Педагогического образования МГУ им. М.В. Ломоносова среди китайских магистрантов 1 курса. Статистические данные доказывают, что информационная грамотность магистрантов и их способность к самостоятельной научно-исследовательской деятельности могут быть значительно улучшены за счет индивидуализированного обучения, осуществляемого под руководством сотрудника библиотеки с помощью КИБР [20].

Были определены организационно-педагогические условия для эффективной реализации авторской технологии в образовательном процессе магистратуры:

- образовательный процесс в магистратуре должен быть технологичным и индивидуализированным во внеаудиторное время, создавая психологически комфортное пространство учения для каждого зарубежного магистранта;

- библиотеки образовательных организаций должны быть оснащены современными информационными ресурсами, необходимыми для поиска, переработки, хранения и использования профессионально значимой научной информации зарубежными магистрантами для исследовательской деятельности;

- следует использовать комплекс информационных библиотечных ресурсов, позволяющий зарубеж- ным магистрантам индивидуально развивать умения и навыки самостоятельной поисково-исследовательской деятельности во внеаудиторное время и овладевать различными информационными навыками;

- сотрудник библиотеки должен осуществлять информационную поддержку научно-исследовательской деятельности каждого зарубежного магистранта во внеаудиторное время, выступая индивидуальным тьютором.

Таким образом, теоретически обоснованная и смоделированная технология внеаудиторной индивидуализации подготовки зарубежных магистров управления образованием под руководством сотрудника библиотеки с помощью комплекса информационных библиотечных ресурсов обеспечивает магистрантам информационную, методическую поддержку и содействует повышению качества их научно-исследовательской деятельности, реализуемой в виртуальном образовательном пространстве библиотек.

\section{Список литературы:}

1. Осмоловская И.М. Организация дифференцированного обучения в современной общеобразовательной школе. Воронеж: МОДЭК, 2005. 214 с.

2. Рыблова А.Н. Технология управления образовательным процессом в системе непрерывного образования. Саратов: Саратовский источник, 2009. 96 с.

3. Селевко Г.К. Энциклопедии образовательных технологий [в 2 Т.]. М.: НИИ шк. технологий, 2006. $401 \mathrm{c}$.

4. Унт И.Э. Индивидуализация и дифференциация обучения. М.: Педагогика, 1990. 192 с.

5. Ковалёва Т.М. Основы тьюторского сопровождения в общем образовании. М.: МПГУ, 2010. 54 с.

6. Зарипова Е.И. Анализ опыта современных российских вузов по созданию индивидуальной образовательной программы магистранта // Вестник Омского государственного педагогического университета. Гуманитарные исследования. 2015. № 5 (9). С. 70-72.

7. Лю Сяньцзюнь. Анализ персонализированного образования в университетах // Журнал высшего образования. 2011. № 3. С. 1-9.

8. Benjamin Bloom. Taxonomy of Educational Objectives: The Classification of Educational Goals. Philadelphia: David McKay Publications, 1956. 216 c.

9. Norman E. Gronlund. Measurement and evaluation in teaching (5th edition). London: Macmillan Publishers, $1985.540 \mathrm{c}$.

10. Рыблова А.Н. Разноуровневое управление иноязычным образовательным процессом подготовки переводчиков: традиции и новации. М.: ФГБОУ ВО «МГЛУ», 2019. 212 c.

11. Пи Ляньшен. Педагогическое проектирование: теория и технология психологии. Пекин: Издательство Высшего Образования, 2000. 229 с.

12. Пи Ляньшен, Цай Вэйцзин. Анализ измерения и оценки результатов обучения на основе ориентированных целей // Вестник Восточно-Китайского педагогического университета. Серия: Педагогические науки. 2000. № 2. С. 40-49.

13. Ву Фати. Анализ сетевой среды обучения на основе ориентированных целей // Исследование электронного образования. 2013. № 7. С. 40-46.

14. Рыблова А.Н., Кан Ц. Проблемы подготовки китайских магистров управления в системе высшего образования // Вестник Московского университета. Серия 20: Педагогическое образование. 2017. № 1. C. 29-35. 
15. Рыблова А.Н. Технология разноуровнего управления иноязычным образовательным процессом в российском университете // Вестник Московского университета. Серия 20: Педагогическое образование. 2018. № 2. С. 78-88.

16. Кан Ц. Методологические основы применения информационных библиотечных ресурсов для индивидуализации обучения в магистратуре // Вестник МГЛУ. Серия «Образование и педагогические науки». 2018. № 1 (790). С. 54-65.

17. Ху Биньу. Стиль обучения и выбор стратегий обучения // Шанхайские образовательные исследования. 1996. № 9. С. 38-39.

\section{TECHNOLOGICAL PROCESS OF EXTRACURRICULAR INDIVIDUALIZATION OF FOREIGN MASTERS TRAINING IN EDUCATION MANAGEMENT}

\section{(C) 2019}

Ryblova Alla Nikolaevna, doctor of pedagogical sciences, professor of Foreign Language Teaching Department Moscow State Linguistic University (Moscow, Russian Federation)

Kang Qiongqiong, postgraduate student of Faculty of Pedagogical Education Lomonosov Moscow State University (Moscow, Russian Federation)

Abstract. The paper poses and solves the problem of the technological organization of extracurricular individual activities of foreign undergraduates. The technological effectiveness of the individualized educational process during graduate studies, developing on the basis of the implementation of the capabilities of information and telecommunication technologies, provides faster and better mastery of knowledge, skills and abilities by foreign undergraduates. Information library resources play a significant role in their development, offer great didactic opportunities for individualized training of foreign Masters in education management who do not speak Russian well and, as a result, need tutorial support outside the classroom to process professionally significant scientific information. The authors present a theoretical and methodological substantiation and the results of modeling and testing the technology of extracurricular individualization of foreign Masters training in education management under the guidance of a librarian using a set of information library resources. This technology provides for the interconnected educational and research activities of a foreign undergraduate and the advisory activities of a librarian, implemented during extracurricular times and aimed at the individual processing of professionally significant scientific information in professionally defined situations using a set of information library resources used as means of pedagogical communication to achieve professional goals. The complex of information library resources includes: print collections, electronic catalogs, search platforms and electronic databases and takes into account the direction of training of foreign undergraduates. The technological process of extracurricular individualization provides an algorithm for the interrelated actions of a librarian and a foreign undergraduate in the design, organization, implementation, monitoring, evaluation and diagnosis of educational results and difficulties of extracurricular individual scientific activity. The organizational and pedagogical conditions for the effective implementation of the authors' technology are determined and diagnostic tools are developed, including tests and diagnostic criteria for the level of mastery of complex of information library resources. The simulated technology provides individual informational, methodological support to each foreign undergraduate and improves the quality of their research activities, implemented in the virtual educational space of libraries.

Keywords: technological process; training of foreign Masters in education management; extracurricular individualization technology; complex of information library resources; librarian; individual control.

\section{ГИМНАСТИКА ЙОГОВ И ВЫСШЕЕ ОБРАЗОВАНИЕ: ПЕДАГОГИЧЕСКИЙ ЭКСПЕРИМЕНТ} (C) 2019

Серпер Сергей Александрович, кандидат экономических наук, докторант

Самарский государственный соџиально-педагогический университет (2. Самара, Российская Федерация)

Аннотащия. Развитие новых направлений как в спорте, физической культуре, так и в сфере преподавания физкультуры - актуальная задача современной педагогики. На современном этапе развития образования и науки важно, чтобы государственные программы сочетались с требованиями российского общества, поэтому всё большее внимание педагоги и методисты уделяют такому направлению оздоровительной политики, как йога. Данное направление нацелено не только на выполнение задач, стоящих перед высшей школой, но и на реализацию государственных программ по развитию здоровья нации. Научно-педагогическое изучение йоги как элемента спортивного образования находится в настоящее время в начальной стадии. Поэтому информация, представленная в статье, имеет уникальный характер: на основании проведённого педагогического экс- 